\title{
An anisotropic turbulent model for solar coronal heating
}

\author{
B. Bigot ${ }^{1,2}$, S. Galtier ${ }^{1,3}$, and H. Politano ${ }^{2}$ \\ 1 Institut d'Astrophysique Spatiale, Bâtiment 121, Université Paris-Sud XI, UMR 8617, 91405 Orsay, France \\ e-mail: bbigot@oca.eu \\ ${ }^{2}$ Université de Nice-Sophia Antipolis, CNRS UMR 6202, Observatoire de la Côte d'Azur, BP 42229, 06304 Nice Cedex 4, France \\ 3 Institut Universitaire de France
}

Received 10 December 2007 / Accepted 26 July 2008

\section{ABSTRACT}

\begin{abstract}
Context. We present a self-consistent model of solar coronal heating in which we include the dynamical effect of the background magnetic field along a coronal structure by using exact results from wave MHD turbulence.

Aims. We evaluate the heating rate and the microturbulent velocity for comparison with observations in the quiet corona, active regions and also coronal holes.

Methods. The coronal structures are assumed to be in a turbulent state maintained by the slow erratic motion of the magnetic footpoints. A description of the large-scale and the unresolved small-scale dynamics are given separately. From the latter, we compute exactly (or numerically for coronal holes) turbulent viscosites used in the former to self-consistently close the system and derive the heating flux expression.

Results. We show that the heating rate and the turbulent velocity compare favorably with coronal observations.

Conclusions. Although the Alfvén wave turbulence regime is strongly anisotropic, and could reduce a priori the heating efficiency, it provides an unexpected, satisfactory model of coronal heating for both magnetic loops and open magnetic field lines.
\end{abstract}

Key words. magnetohydrodynamics (MHD) - Sun: corona - turbulence

\section{Introduction}

Information about the solar corona from spacecraft missions like Yohkoh, SoHO (Solar \& Heliospheric Observatory) or TRACE (Transition Region And Coronal Explorer) launched in the 1990s reveals a very dynamical and complex medium structured in a network of magnetic field lines. These observations clearly demonstrated the fundamental role of the magnetic field on the plasma dynamics in the solar atmosphere. The solar corona contains a variety of structures over a broad range of scales from about $10^{5} \mathrm{~km}$ until the limit of resolution (about one arcsec). It is very likely that structures at much smaller scales exist but have not yet been detected. The new spacecrafts STEREO, Hinode or SDO (Solar Dynamics Observatory) will help our understanding of the small-scale nature of the corona.

Observations in UV and X-ray show a solar corona that is extremely hot with temperatures exceeding $10^{6} \mathrm{~K}$ - close to hundred times the solar surface temperature. These coronal temperatures are highly inhomogeneous: in the quiet corona much of the plasma lies near $1-2 \times 10^{6} \mathrm{~K}$ and $1-8 \times 10^{6} \mathrm{~K}$ in active regions. Then, one of the major questions in solar physics concerns the origin of such high values of coronal temperature. The energy available in the photosphere is clearly sufficient to supply the total coronal losses (Withbroe \& Noyes 1977) which is estimated to be $10^{4} \mathrm{~J} \mathrm{~m}^{-2} \mathrm{~s}^{-1}$ for active regions and about one or two orders of magnitude smaller for the quiet corona and coronal holes. The main issue is thus to understand how the available photospheric energy is transferred and accumulated in the solar corona, and by what processes it is dissipated.

It is widely believed that the energy input comes from the slow random motion of the convective layer below the photosphere, but the mechanisms that heat the solar corona remain controversial. Heating models are often classified into two categories: "AC" and "DC" heating. "AC" heating by Alfvén waves was suggested for the first time by Alfvén (1947). In nonuniform plasmas, this heating mechanism could be sustained by the resonant absorption of Alfvén waves (Hollweg 1984), or by phase mixing (Heyvaerts \& Priest 1983). The necessary condition for "AC" heating is that the characteristic time of motion excitation has to be shorter than the characteristic time of wave propagation across the coronal loops. In the opposite situation, the most efficient heating is the "DC" heating by direct current. In this case, the quasi-static energy input allows the accumulation of magnetic energy, the generation of currents and finally heating, for example, by reconnection of magnetic field lines (Priest \& Forbes 2000) or by resistive dissipation of current sheets. However, in this simple version, these mechanisms are not efficient enough to explain coronal heating. Additional processes are thus generally introduced like turbulence, which can generate small scales where dissipation is much more efficient (see, e.g., Gomez \& Ferro Fontan 1988, 1992; Heyvaerts \& Priest 1992; Einaudi et al. 1996; Dmitruk et al. 1997; Galtier \& Pouquet 1998; Galtier \& Bhattacharjee 2003; Buchlin et al. 2007). Moreover, turbulence could explain the measurements of nonthermal velocities revealed by the width of EUV and FUV lines. Observations of the transition region and corona of the quiet Sun from SUMER onboard SoHO reveal nonthermal velocities of about $30 \mathrm{~km} \mathrm{~s}^{-1}$ for temperatures around $3 \times 10^{5} \mathrm{~K}$ with a peak up to $55 \mathrm{~km} \mathrm{~s}^{-1}$ for some S IV lines (Warren et al. 1997; Chae et al. 1998).

Many observations of the solar atmosphere tend to show plasma in a turbulent state with a Reynolds number evaluated at about $10^{12}$. In particular, the most recent Hinode pictures seem to show a magnetic field controlled by plasma turbulence at all 


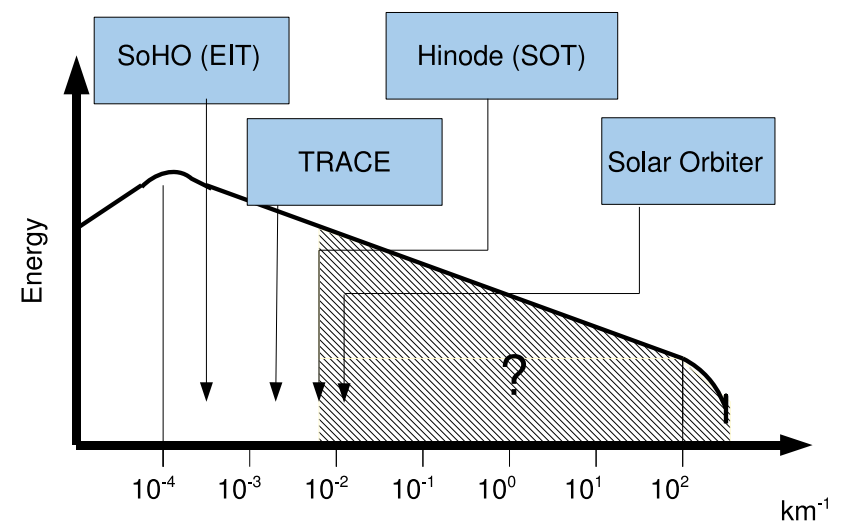

Fig. 1. Schematic view of the energy spectrum of the coronal plasma in logarithmic coordinates. The spatial resolution of different spacecraft instruments is reported: EIT/SoHO (1850 km), TRACE $(350 \mathrm{~km})$, SOT/Hinode $(150 \mathrm{~km})$ and Solar Orbiter $(35 \mathrm{~km})$. The gray zone indicates currently unresolved small-scales which will be modeled by turbulent viscosities.

scales (Nature 2007; see, also, Doschek et al. 2007). Thus, the turbulent activity of the corona is one of the key issues to understand the heating processes. In the framework of turbulence, the energy supplied by the photospheric motion and transported by Alfvén waves through the corona is transferred towards smaller and smaller scales by nonlinear coupling between modes (the socalled energy cascade) until dissipative scales are reached from which the energy is converted into heating. The main coronal structures considered in such a scenario are the magnetic loops which cover the solar surface, in active and quiet regions. Each loop is basically an anisotropic bipolar structure anchored in the photosphere. It forms a tube - or an arcade - of magnetic fields in which the dense and hot matter is confined. Because a strong guiding magnetic field $\left(\boldsymbol{B}_{0}\right)$ is present, the nonlinear cascade that occurs is strongly anisotropic with small scales mainly developed in the $\boldsymbol{B}_{0}$ transverse planes. In Fig. 1, we present a schematic view of the turbulent energy spectrum expected for the coronal plasma which follows a power law over several decades. The larger scales may be determined directly by observation and correspond to about $10^{5} \mathrm{~km}$. The inertial range - that determines the range of scales where the turbulent cascade operates roughly starts between $10^{4} \mathrm{~km}$ and $10^{3} \mathrm{~km}$, and extends down to unresolved (by spacecraft observations) scales which may be estimated, from dimensional analysis, as of a few meters. The spatial resolution of different instruments is also reported to show the gap that we need to fill in order to completely resolve the heating processes that occur at the smallest dissipative scales.

The aim of the present study is coronal heating to perform of modeling by magnetohydrodynamic (MHD) turbulence. We explain the connection between the large scales (at which energy is injected at loop footpoints through photospheric motion) and the smallest scales (at which energy is dissipated and converted into heat). The input energy propagates through the network of magnetic field lines by "inward" and "outward" Alfvén waves which nonlinearly interact and produce a turbulent cascade towards small scales. The foundation of our model is the one originally proposed by Heyvaerts \& Priest (1992) where the unresolved small-scales are modeled through turbulent viscosities. These viscosities were extracted from an ad hoc EDQNM (EddyDamped Quasi-Normal Markovian) closure model of MHD turbulence developed in spectral space for isotropic flows (Pouquet et al. 1976). Spacecraft missions like SoHO, TRACE, or more recently Hinode show that this assumption is clearly not adapted

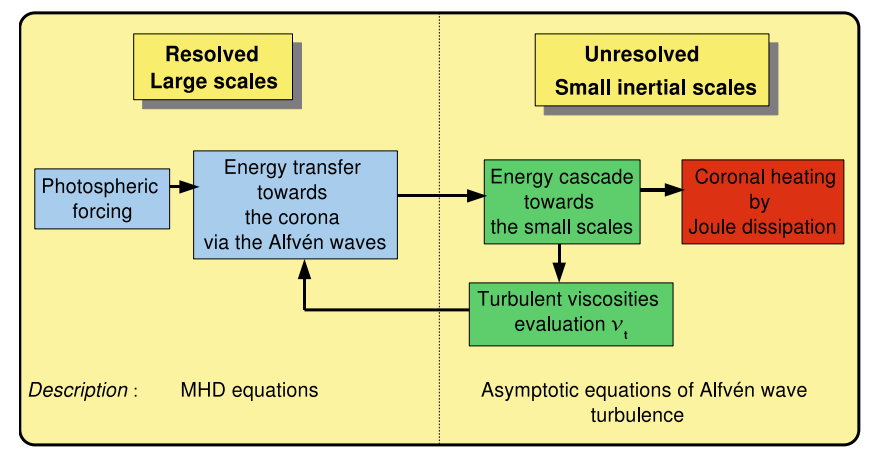

Fig. 2. Schematic view of the self-consistent heating model. The resolved large-scales coronal flow is described by the "large-scale" MHD equations where turbulent (instead of standard) viscosity and resistivity are used to determine the inertial small-scale nonlinear dynamics. From these equations we compute the energy flux function released by the photospheric motion. The energy is transported by Alfvén waves through the corona and the nonlinear interactions between wave packets generate an inertial cascade, i.e. a production of smaller and smaller scales, that finally reach the dissipative smallest scales from which the energy is converted into ohmic dissipation. The unresolved inertial small-scales are described by the asymptotic equations of Alfvén wave turbulence from which the turbulent transport coefficients are computed. A self-consistent model is finally obtained by introducing the expression of these turbulent viscosities into the energy flux function. Then, the energy flux function is entirely determined and the heating rate can be evaluated.

to magnetic loops which are characterized by a strong longitudinal mean field $\boldsymbol{B}_{0}$, compared to the magnetic perpendicular components, whose anisotropic nonlinear effects on the turbulent plasmas dynamics are more than likely, as many numerical simulations have shown. For that reason, in the present model, we use turbulent viscosities computed from an asymptotic (exact) closure model of MHD turbulence (Galtier et al. 2000, 2002), also called Alfvén wave turbulence. Signatures of such a regime have been detected in the middle magnetosphere of Jupiter (Saur et al. 2002). In our turbulent heating model, the unresolved smallscale equations are perturbatively developed around a strong magnetic field $\boldsymbol{B}_{0}$ which leads to a strongly anisotropic turbulence. From the derived equations, we compute the turbulent viscosities which eventually allow us to obtain a self-consistent free-parameter model of coronal heating from which we predict a heating rate and a turbulent velocity that favorably compare with observations. Figure 2 summarizes the schematic algorithm followed to obtain a self-consistent heating model.

The organization of the paper is as follows: the next Section is dedicated to the large-scale description of magnetic loops where, in particular, we rederive some main results obtained by Heyvaerts \& Priest (1992), using a more adapted notation. Then, in Sect. 3, the small-scale description is given and the turbulent viscosities are obtained. The model predictions (heating rate and turbulent velocity) for magnetic loops in active regions and in the quiet corona are given in Sect. 4. They are generalized to open magnetic lines in coronal holes in Sect. 5. The last section is devoted to the discussion and conclusion.

\section{Large-scale description of magnetic loops}

\subsection{Geometry and boundary conditions}

We consider a set of magnetic loops - an arcade - anchored in the photosphere and subjected to the erratic motion of convective cells. For simplicity, this arcade has a rectangular cross surface 


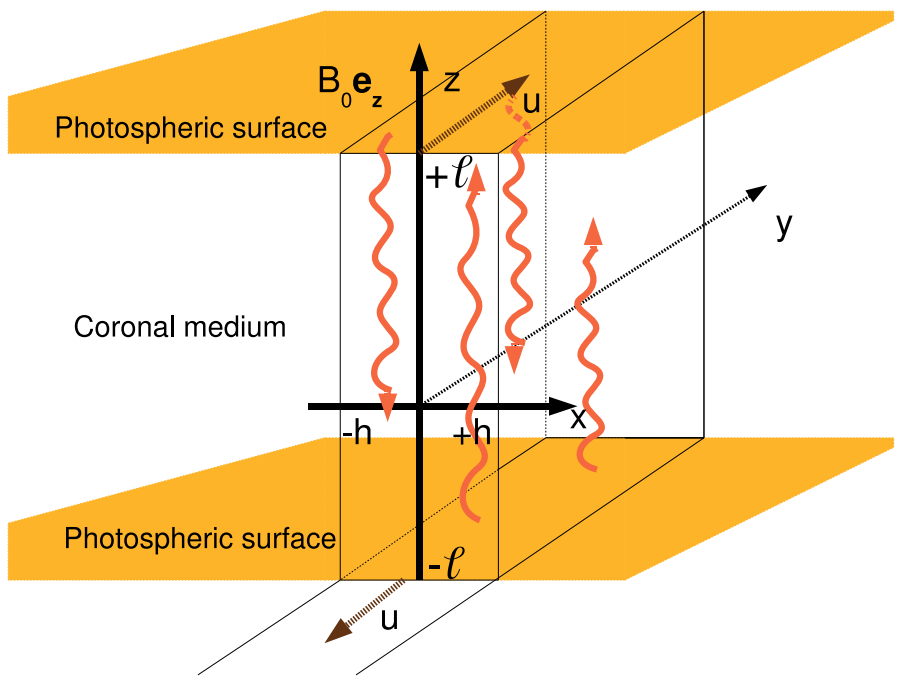

Fig. 3. Arcade geometry: the coronal plasma is confined in a volume delimited by $-h<x<+h,-\infty<y<+\infty$ and $-\ell<z<+\ell$. The photosphere appears as two boundary planes at altitudes $z=-\ell$ and $z=+\ell$. Note the presence of Alfvén waves propagating in opposite directions along $\boldsymbol{B}_{0}$.

which defines the perpendicular directions and it is elongated along the longitudinal magnetic field $\boldsymbol{B}_{0}=B_{0} \boldsymbol{e}_{z}$ that defines the parallel direction as shown in Fig. 3. This magnetic structure is filled by a plasma which will be modeled as an incompressible MHD fluid. In the first part of this paper, we are concerned with magnetic loops; later on we will extend our results to open magnetic field lines for which the boundary conditions will be modified. For a magnetic arcade, the boundary limits are made of two planes at altitudes $z=-\ell$ and $z=+\ell$ which are connected by the uniform magnetic field $\boldsymbol{B}_{0}$. Thus, the length of the loop in the longitudinal direction is $2 \ell$. The cross surface is delimited as $-h<x<+h$ and $-\infty<y<+\infty$. In other words, we assume an arcade characterized by a thickness of $2 h$, where $h$ is the coherence length of the magnetic field at the base of the corona, and with a large depth compare to the other length scales, with a translational invariance assumed in the $y$-direction. Note that we do not treat the expansion of the magnetic field at the footpoint loops where $B_{0}$ has to be seen as an average value of the field at the base of the corona rather than in the photosphere itself.

The energy reservoir for the solar corona resides in the subphotospheric convective layer. Indeed, the high- $\beta$ plasma in the photosphere constrains the magnetic field lines to be convected by the plasma flow. These motions eventually induce a shearing and a torsion of coronal magnetic field lines. The boundary motions at footpoint loops are assumed to be perpendicular and independent of the coronal plasma. More precisely, we assume for the boundary velocity field parallel to the $y$-axis:

$\boldsymbol{u}(x, z=\ell)=-\boldsymbol{u}(x, z=-\ell)=U(x) \boldsymbol{e}_{y}$.

This input of energy is then propagated through the coronal medium where the low- $\beta$ confines the plasma into the magnetic arcade. These boundary motions are then able to produce and maintain a state of full turbulence in the corona. Note finally the absence of mass flow through the boundaries.

\subsection{The MHD model}

The standard incompressible MHD equations are used as a first approximation to model the solar plasma, namely:

$$
\begin{aligned}
& \partial_{\mathrm{t}} \boldsymbol{u}+\boldsymbol{u} \cdot \boldsymbol{\nabla} \boldsymbol{u}=-\nabla P / \rho_{0}+\boldsymbol{j} \times \boldsymbol{B} / \rho_{0}+v \Delta \boldsymbol{u}, \\
& \nabla \cdot \boldsymbol{u}=0, \\
& \partial_{\mathrm{t}} \boldsymbol{B}=\boldsymbol{\nabla} \times(\boldsymbol{u} \times \boldsymbol{B})+\eta \Delta \boldsymbol{B}, \\
& \boldsymbol{\nabla} \cdot \boldsymbol{B}=0
\end{aligned}
$$

$\partial_{\mathrm{t}}\left(\rho_{0} \frac{u^{2}}{2}+\frac{B^{2}}{2 \mu_{0}}\right)+\boldsymbol{\nabla} \cdot \mathcal{F}=\mathcal{S}-\mathcal{D}$,

$\mathcal{F}=\boldsymbol{u} \cdot\left(\rho_{0} \frac{u^{2}}{2}+P-\overline{\bar{\sigma}}\right)+\frac{\boldsymbol{E} \times \boldsymbol{B}}{\mu_{0}}$,

$\boldsymbol{E}+\boldsymbol{u} \times \boldsymbol{B}=\mu_{0} \eta \boldsymbol{j}$,

where $\boldsymbol{u}$ is the velocity, $\boldsymbol{B}$ the magnetic field (such as $\boldsymbol{B}=\boldsymbol{B}_{0}+\boldsymbol{b}$ ), $\boldsymbol{j}=\boldsymbol{\nabla} \times \boldsymbol{b} / \mu_{0}$ the current density (where $\mu_{0}$ is the permeability of vacuum), $\boldsymbol{E}$ the electric field, $\overline{\bar{\sigma}}$ the viscous stress tensor (with $\left.\sigma_{i j}=\rho_{0} v\left(\partial u_{i} / \partial r_{j}+\partial u_{j} / \partial r_{i}\right)\right), v$ the kinematic viscosity, $\eta$ the magnetic diffusivity and $\rho_{0}$ the uniform mass density, and $P$ is the gas pressure. In the energy Eq. (6), the right hand side term $\mathcal{S}$ is the large-scale energy input created by the photospheric motion and $\mathcal{D}$ is the dissipation which mainly happens at the smallest scales because of, e.g., viscous and resistive effects. The left hand side term, $\boldsymbol{\nabla} \cdot \mathcal{F}$, denotes the nonlinear transfer of total (kinetic plus magnetic) energy. (Note that all terms in Eq. (6) are expressed in unit volume.) Equation (7) describes how the total energy is nonlinearly transferred at different scales by velocity transport, by work due to the gas pressure, the viscous stress or the electromagnetic work due to the Poynting vector $\left(\boldsymbol{E} \times \boldsymbol{B} / \mu_{0}\right)$. Other (higher order) processes like thermal conductivity which happens at microscopic scales are not considered here. Finally, Eq. (8) is the standard Ohm's law which allows us to close the system.

The basic idea of this coronal model is the separation of the non-resolved inertial small-scale dynamics (see Figs. 1 and 2) from the large-scale dynamics detected with current instruments. In practice, we assume that the previous MHD equations describe the large-scale behavior of the plasma; the nonlinear small-scale processes (i.e. the effects of the inertial small-scales on the large-scale dynamics) will be modeled through effective dissipations, also called turbulent viscosity and resistivity. In other words, the usual molecular viscosity and magnetic resistivity are replaced by turbulent coefficients which are several orders of magnitude larger. The expression of these turbulent viscosities (hereafter denoted $v_{\mathrm{t}}$ and $\eta_{\mathrm{t}}$ ) will be derived in Sect. 3 and the MHD equations in the next section.

An important assumption to derive this heating model is that the slow boundary motions are able to sustain a fully turbulent state in the corona, with a typical photospheric time longer than the coronal time scales. Basically, this statement means that the characteristic time of the photosphere has to be longer than the time needed for a perturbation to cross the coronal loops. For a typical longitudinal direction $\ell$ of $10^{7} \mathrm{~m}$ and an Alfvén speed of several $10^{6} \mathrm{~m} / \mathrm{s}$, one finds a crossing time of the order of $1 \mathrm{~s}$. For a photospheric granule with a size $\lambda=10^{6} \mathrm{~m}$ and a velocity $\bar{u}=10^{3} \mathrm{~m} / \mathrm{s}$, one finds a lifetime of about $10^{3} \mathrm{~s}$. In this case, the time scale separation is thus well satisfied. However, a validity condition may arise if the turbulent nature of the photospheric motion is taken into account since their velocities seem to follow a Kolmogorov spectrum (Roudier et al. 1987; Chou et al. 1991; 
Espagnet et al. 1993). Then the photospheric velocity at a given scale $\lambda$ should scale as $\lambda^{1 / 3}$ and the typical photospheric nonlinear time as $\lambda^{2 / 3}$, which decreases with the size of the granules. The associated condition is discussed and used in Sect. 2.8.

\subsection{Large-scale solutions}

The nonlinear solutions of the previous MHD equations are non trivial and require direct numerical simulations on supercomputers. However, we are here mainly interested in the largescale behavior of the coronal flow which allows us to make some simplifications. We will seek solutions of the form

$$
\begin{aligned}
\boldsymbol{u} & =u(x, z) \boldsymbol{e}_{y}, \\
\boldsymbol{B} & =B_{0} \boldsymbol{e}_{z}+b(x, z) \boldsymbol{e}_{y},
\end{aligned}
$$

independent of $y$ and $t$, which satisfy, in particular, the divergence free conditions (3) and (5). These solutions do not depend on time since we assume magnetic loops maintained in a stationary regime of turbulence. We also observe that the fluctuating velocity and magnetic fields are taken perpendicular to the uniform magnetic field $\boldsymbol{B}_{0}$. This assumption is compatible with the fact that, in strongly magnetized media, pseudo-Alfvén waves are mainly along the uniform magnetic field whereas the nonlinear dynamics is dominated by shear-Alfvén waves in the transverse planes. We recall that shear-Alfvén and pseudo-Alfvén waves are the two kinds of linear perturbations about the equilibrium, the latter being the incompressible limit of slow magnetosonic waves. Replacing the previous solution types into the MHD Eqs. (2) and (4) leads to

$$
\begin{aligned}
\partial_{x}\left(P+b^{2}(x, z) / 2 \mu_{0}\right) & =0, \\
\partial_{z}\left(P+b^{2}(x, z) / 2 \mu_{0}\right) & =0, \\
B_{0} \partial_{z} b(x, z)+\mu_{0} \rho_{0} v_{\mathrm{t}} \Delta u(x, z) & =0, \\
B_{0} \partial_{z} u(x, z)+\eta_{\mathrm{t}} \Delta b(x, z) & =0 .
\end{aligned}
$$

These equations describe the large-scale evolution of the plasma once the turbulent regime is established. In particular, they allow us to calculate the pressure field when the magnetic field is known. The linear form of Eqs. (13) and (14) may be misleading since the small-scale nonlinearities are concentrated in the turbulent coefficient transports, namely the turbulent eddy viscosity $v_{\mathrm{t}}$ and magnetic diffusivity $\eta_{\mathrm{t}}$. This assumption can be justified when looking at the nonlinear evolution equations for the energy spectra, as used in Sect. 3. Indeed, in Fourier space, a nonlocal analysis leads to diffusive effects due to the smallscale dynamics over the large scale motions, actually stemming from absorption terms involved in the modeled energy transfers between small and large scales.

\subsection{Photospheric conditions}

The photospheric forcing introduced in Eq. (1) mimics the largescale motion of magnetic footpoints imposed by the convective cells. Following Heyvaerts \& Priest (1992), we assume that the frozen-in law is satisfied in the dense photosphere which leads, when coupled with the Ohm's law (8), to the boundary relation for the electric field:

$\boldsymbol{E}(x, z= \pm \ell)=\mp U(x) B_{0} \boldsymbol{e}_{x}$.

In the close neighborhood of the photosphere, this relation is modified to take into account the magnetic diffusivity. This gives for the $x$-component:

$E_{x}(x, z \simeq \pm \ell)=\mp \eta_{\mathrm{t}} \frac{\partial b}{\partial z}(x, z) \mp U(x) B_{0}$.
The continuity of the tangential component of the electric field implies that

$\frac{\partial b}{\partial z}(x, z= \pm \ell)=0$

These photospheric conditions are of course compatible with Eqs. (13) and (14) when $z= \pm \ell$.

\subsection{Energy flux injection}

The aim of this section is to express the energy flux released by photospheric motion along coronal loops. We are thus interested in the $z$-component of the flux (7) at the boundaries $z= \pm \ell$, namely:

$\mathcal{F}_{z}(x, z= \pm \ell)=\boldsymbol{e}_{z} \cdot\left[\boldsymbol{u} \cdot\left(\rho_{0} \frac{u^{2}}{2}+P-\overline{\bar{\sigma}}\right)+\frac{\boldsymbol{E} \times \boldsymbol{B}}{\mu_{0}}\right]$

The velocity boundary conditions (1) do not involve (convective) mass flows through the photospheric planes since $u_{z}(z= \pm l)=0$. Therefore, the two first terms in expression (18) do not contribute, and the input flux reduces to

$\mathcal{F}_{z}(x, z= \pm l)=\Pi_{z}+\mathcal{P}_{z}$

with

$\Pi_{z}=-(\boldsymbol{u} \cdot \overline{\bar{\sigma}})_{z}, \mathcal{P}_{z}=\left(\frac{\boldsymbol{E} \times \boldsymbol{B}}{\mu_{0}}\right)_{z}$,

i.e. only the viscous stress tensor and the Poynting vector contribute to the energy flux transfer to the corona. One of the main goal of this paper is to evaluate this energy flux by first solving Eqs. (13) and (14), which depend on the turbulent viscosity and resistivity, and second, by calculating these turbulent coefficients from Alfvén wave kinetic equations.

\subsection{Solving the large-scale dynamics}

In this section, we solve Eqs. (13) and (14) to find the large-scale plasma behavior. We develop the $x$-dependence of the velocity and magnetic fields in Fourier series (since $h$ is assumed to be the coherence length of the magnetic and velocity fields in the $x$-direction)

$\xi(x, z)=\sum_{n=1}^{+\infty} \xi_{n}^{(1)}(z) \cos \frac{n \pi x}{h}+\sum_{n=1}^{+\infty} \xi_{n}^{(2)}(z) \sin \frac{n \pi x}{h}$,

where $\xi$ is either $u$ or $b$. Note that other solutions are possible with, e.g. a twisted flux tube (Inverarity \& Priest 1995), which do not drastically change the coronal heating predictions. Substitution of (21) into (13) and (14) gives

$$
\begin{array}{r}
B_{0} \frac{\mathrm{d} b_{n}^{(i)}}{\mathrm{d} z}+\mu_{0} \rho_{0} v_{\mathrm{t}}\left(\frac{\mathrm{d}^{2}}{\mathrm{~d} z^{2}}-\frac{n^{2} \pi^{2}}{h^{2}}\right) u_{n}^{(i)}=0, \\
B_{0} \frac{\mathrm{d} u_{n}^{(i)}}{\mathrm{d} z}+\eta_{\mathrm{t}}\left(\frac{\mathrm{d}^{2}}{\mathrm{~d} z^{2}}-\frac{n^{2} \pi^{2}}{h^{2}}\right) b_{n}^{(i)}=0,
\end{array}
$$

where $(i)$ stands for indices (1) or (2), and finally

$$
\frac{\mathrm{d}^{4} u_{n}^{(i)}}{\mathrm{d} z^{4}}-\left[\frac{B_{0}^{2}}{\mu_{0} \rho_{0} v_{\mathrm{t}} \eta_{\mathrm{t}}}+2\left(\frac{n \pi}{h}\right)^{2}\right] \frac{\mathrm{d}^{2} u_{n}^{(i)}}{\mathrm{d} z^{2}}+\left(\frac{n \pi}{h}\right)^{4} u_{n}^{(i)}=0
$$


Note that the magnetic field satisfies the same equation, only the boundary conditions make the distinction between the two fields. Using the following notation:

$\alpha=\frac{B_{0}}{\sqrt{\mu_{0} \rho_{0} v_{\mathrm{t}} \eta_{\mathrm{t}}}}, \alpha \lambda_{n}=\frac{n \pi}{h}, r^{m}=\frac{\mathrm{d}^{m}}{\mathrm{~d}(\alpha z)^{m}}$,

Eq. (24) rewrites

$r^{4} u_{n}^{(i)}-\left(1+2 \lambda_{n}^{2}\right) r^{2} u_{n}^{(i)}+\lambda_{n}^{4} u_{n}^{(i)}=0$,

whose solutions may be expressed in terms of exponentials. The photospheric boundary conditions (1) and (17) combined with Eq. (22) gives the relations

$u_{n}^{(i)}(z= \pm \ell)= \pm U_{n}^{(i)}$ and $\frac{\mathrm{d}^{2} u_{n}^{(i)}}{\mathrm{d} z^{2}}(z= \pm \ell)= \pm \alpha^{2} \lambda_{n}^{2} U_{n}^{(i)}$,

which lead eventually to the solutions

$u_{n}^{(i)}(z)=\frac{U_{n}^{(i)}}{\mathfrak{R}_{n}^{+}+\mathfrak{R}_{n}^{-}}\left[\frac{\mathfrak{R}_{n}^{-} \sinh \left(\mathfrak{R}_{n}^{+} \alpha z\right)}{\sinh \left(\mathfrak{R}_{n}^{+} \alpha \ell\right)}+\frac{\mathfrak{R}_{n}^{+} \sinh \left(\mathfrak{R}_{n}^{-} \alpha z\right)}{\sinh \left(\mathfrak{R}_{n}^{-} \alpha \ell\right)}\right]$,

where

$\mathfrak{R}_{n}^{ \pm}=\frac{\sqrt{1+4 \lambda_{n}^{2}} \pm 1}{2}$

The substitution of (28) into (22) and (23) gives, after some manipulations, the following expression for the magnetic field Fourier coefficients

$b_{n}^{(i)}(z)=\frac{B_{0} U_{n}^{(i)}}{\mathfrak{R}_{n}^{+}+\mathfrak{R}_{n}^{-}}\left(\frac{\mathfrak{R}_{n}^{+} \cosh \left(\mathfrak{R}_{n}^{-} \alpha z\right)}{\eta \alpha \sinh \left(\mathfrak{R}_{n}^{-} \alpha \ell\right)}-\frac{\mathfrak{R}_{n}^{-} \cosh \left(\mathfrak{R}_{n}^{+} \alpha z\right)}{\eta \alpha \sinh \left(\mathfrak{R}_{n}^{+} \alpha \ell\right)}\right)$.

\subsection{Evaluation of the energy flux}

From the solutions (28) and (30) it is now possible to calculate the energy flux (19) at footpoint levels $z= \pm \ell$. The contribution of the Poynting vector to the flux is

$\mathcal{P}_{z}(x, \pm \ell)=\mp \frac{B_{0}}{\mu_{0}} U(x) b(x, z= \pm \ell)$,

and the contribution of the viscous stress tensor is

$\Pi_{z}(x, \pm \ell)=\mp \rho_{0} v_{\mathrm{t}} U(x) \sigma_{z y}=\mp \rho_{0} v_{\mathrm{t}} U(x) \frac{\partial u(x, z= \pm \ell)}{\partial z}$.

The input flux coming from the photospheric boundaries $(z=$ $\pm \ell$ ) is calculated by averaging over the $x$-periodicity of the velocity and magnetic fields (21):

$\left|\mathcal{F}_{z}(z= \pm \ell)\right|=\frac{1}{2 h}\left|\int_{-h}^{+h}\left[\mathcal{P}_{z}(x, \pm \ell)+\Pi_{z}(x, \pm \ell)\right] \mathrm{d} x\right|$.

After some manipulations, we obtain the total flux (from the two footpoint levels):

$$
\begin{aligned}
\left|\mathcal{F}_{z}\right|= & \rho_{0} \sum_{n=1}^{\infty} \sqrt{\frac{\nu_{\mathrm{t}}}{\eta_{\mathrm{t}}}} \frac{B_{0}}{\sqrt{\rho_{0} \mu_{0}}} U_{n}^{2}\left(\frac{\sinh (\alpha \ell)}{\cosh \left[\sqrt{1+4 \lambda_{n}^{2}} \alpha \ell\right]-\cosh (\alpha \ell)}\right. \\
& \left.+\frac{\left(1+2 \lambda_{n}^{2}\right) \sinh \left[\sqrt{1+4 \lambda_{n}^{2}} \alpha \ell\right] / \sqrt{1+4 \lambda_{n}^{2}}}{\cosh \left[\sqrt{1+4 \lambda_{n}^{2}} \alpha \ell\right]-\cosh (\alpha l)}\right)
\end{aligned}
$$

with $U_{n}^{2}=U_{n}^{(1)^{2}}+U_{n}^{(2)^{2}}$. Note the appearance of a turbulent magnetic Prandtl number $\left(v_{\mathrm{t}} / \eta_{\mathrm{t}}\right)$ whose origin is directly linked to the action of nonlinear small-scale processes on large-scales. We will later show that this number may be taken equal to unity in the Alfvén wave turbulence regime. Also note that the turbulent viscosities enter in expression (34) through the coefficients $\alpha$ and $\lambda_{n}$. The origin of the presence of $v_{\mathrm{t}}$ and $\eta_{\mathrm{t}}$ is thus due, on the one hand, to the large-scale MHD Eqs. (13), (14) and, on the other hand, to the Poynting vector (31) and the viscous stress tensor (32) which also include the small-scale nonlinear retroaction. The energy injected from the magnetic footpoints is propagated through the corona by Alfvén waves. Then, the collisions between "upward" and "downward" wave packets lead to nonlinear dynamics which is characterized by a turbulent cascade that transfers energy from the energy injection scales down to the smallest dissipative scales where energy is destroyed, and the corona heated. The coronal loops reach a stationary state of fully developed turbulence when the dissipation equals the electromagnetic and viscous stress.

\subsection{Turbulent spectrum of photospheric velocities}

The coronal heating flux is now evaluated from (34). The turbulent viscosities entering into this flux expression will be computed in the next Section. Therefore, the last unknown is the magnitude of the velocity at the photospheric boundaries (namely $U_{n}$ ). The goal of this section is to evaluate the scale dependence of photospheric velocities.

As reported by different measurements (Roudier et al. 1987; Chou et al. 1991; Espagnet et al. 1993), the photospheric velocities are assumed to follow a Kolmogorov isotropic spectrum

$u^{2}(k)=\frac{2}{3} \bar{u}^{2} k_{i n j}^{2 / 3} k^{-5 / 3}$,

with

$\bar{u}^{2}=\int_{k_{i n j}}^{+\infty} u^{2}(k) \mathrm{d} k=\sum_{n=1}^{+\infty} U_{n}^{2} / 2$

where $\bar{u}$ is the photospheric rms velocity, $U_{n}^{2}$ is the power spectrum of the boundary velocity fluctuations, and $k_{\text {inj }}$ is the largest scale at which energy is injected into the system; it corresponds to the size of the largest granular cells, i.e. about $1000 \mathrm{~km}$. In other words, it is the integral scale of the turbulent photospheric flow from which the inertial range starts. The wave number $k_{i n j}$ may be directly connected to the thickness of the arcade, such that $k_{i n j}=\pi / h$. More generally, the wave number $k$ and the harmonic number $n$ may be connected through the relation $k=n \pi / h$. Converting the sum in Eq. (36) into an integral gives the relation

$U_{n}^{2}=\frac{2 \pi}{h} u^{2}(k)=\frac{4}{3} \bar{u}^{2} n^{-5 / 3}$

The basic idea of the model is that Alfvén wave packets interact nonlinearly along coronal magnetic structures in which the plasma is assumed to be maintained in a fully turbulent state because of the slow photospheric motion. We have seen in Sect. 2.2 that a condition may arise on time-scales since the photosphere is also turbulent and its typical nonlinear time, $\tau_{\mathrm{nl}}^{\mathrm{ph}}$, may be very short for small granules. In order to satisfy this condition, we impose that $\tau_{\mathrm{nl}}^{\mathrm{ph}}(\lambda)>\tau_{\text {cross }}$, where $\tau_{\text {cross }}$ is the time needed for a perturbation to cross half of the loops of length $2 \ell$ at speed $B_{0} / \sqrt{\rho_{0} \mu_{0}}$, namely

$\tau_{\text {cross }} \sim \frac{\ell \sqrt{\rho_{0} \mu_{0}}}{B_{0}}$ 
and $\tau_{\mathrm{nl}}^{\mathrm{ph}}(\lambda)$ is the eddy turnover (nonlinear) time of granular structures at a typical scale $\lambda$ and velocity $u(\lambda)$;

$\tau_{\mathrm{nl}}^{\mathrm{ph}}(\lambda) \sim \frac{\lambda}{u(\lambda)} \sim \frac{\lambda^{2 / 3}}{\bar{u}}\left(\frac{2 \pi}{k_{\text {inj }}}\right)^{1 / 3}$.

This time-scale separation induces for the wave number $k=$ $2 \pi / \lambda$, the inequality $k<k_{\text {cross }}$, where

$k_{\text {cross }}=\frac{\pi}{h} N_{\text {cross }}$, with $N_{\text {cross }}=\left(2 \frac{h}{\ell} \frac{B_{0} / \sqrt{\rho_{0} \mu_{0}}}{\bar{u}}\right)^{3 / 2}$.

Hence, the upper limit of the summation on harmonic numbers in (34) is given by $N_{\text {cross }}$ which is a large number since the Alfvén velocity is much larger than the photospheric rms velocity. For that reason, the expression (34) may also be written as an integral with

$U^{2}(k)=\frac{4}{3}\left(\frac{\pi}{h}\right)^{5 / 3} \bar{u}^{2} k^{-5 / 3}$

The energy injection produced by the photospheric motion is finally rewritten as follows:

$\left|\mathcal{F}_{z}\right|=\rho_{0} \int_{k_{\text {inj }}}^{k_{\text {cross }}} \sqrt{\frac{\nu_{\mathrm{t}}}{\eta_{\mathrm{t}}}} \frac{B_{0}}{\sqrt{\rho_{0} \mu_{0}}} U^{2}(k) \Psi(k) \frac{h}{\pi} \mathrm{d} k$,

with

$$
\begin{aligned}
\Psi(k)= & \frac{\sinh (\alpha \ell)}{\cosh \left[\sqrt{\alpha^{2}+4 k^{2}} \ell\right]-\cosh (\alpha \ell)} \\
& +\frac{\alpha^{2}+2 k^{2}}{\alpha \sqrt{\alpha^{2}+4 k^{2}}} \frac{\sinh \left[\sqrt{\alpha^{2}+4 k^{2}} \ell\right]}{\cosh \left[\sqrt{\alpha^{2}+4 k^{2}} \ell\right]-\cosh (\alpha \ell)} .
\end{aligned}
$$

Under the time-scale condition discussed above, the coronal structure is submitted to slow external forcing. Note that other time scales are present in our problem; namely, the nonlinear coronal time, $\tau_{\mathrm{nl}}$, and the Alfvén time, $\tau_{\mathrm{A}}$, which has nothing to do with the crossing time $\tau_{\text {cross }}$ introduced above. It may be interpreted as the time of interaction between two counterpropagating Alfvén wave packets. Both times will be used in Sect. 3 where the nonlinear small-scale dynamics will be analyzed. The main assumption made in such wave turbulence dynamics is that the Alfvén time is smaller than the nonlinear coronal time. Note that in MHD the transfer time-scale is built on $\tau_{\mathrm{nl}}$ and $\tau_{\mathrm{A}}$ which makes a great difference in Navier-Stokes fluids for which the transfer time is the nonlinear time. This point will be further discussed in the next section. Then the following hierarchy of times may be estimated

$\tau_{\mathrm{A}}<\tau_{\mathrm{nl}}<\tau_{\mathrm{cross}}<\tau_{\mathrm{nl}}^{\mathrm{ph}}$.

As seen in Sect. 2.2, the nonlinear photospheric time can be as large as $10^{3} \mathrm{~s}$ and the crossing time is about $1 \mathrm{~s}$. For the two other characteristic time scales, no observational constraints can be used. However, recent direct numerical simulations (Bigot et al. 2008b) show clearly that a turbulent MHD flow evolving in a strong uniform magnetic field $\left(B_{0}>b\right)$ exhibits a time ratio $\tau_{\mathrm{A}} / \tau_{\mathrm{nl}}<1$, which decreases as the strength of the uniform field increases.

\section{Small-scale dynamics and anisotropy}

In the previous section, we have been able to evaluate the flux of energy released by photospheric motion along coronal loops. The calculation has been greatly simplified by the assumption of a scale separation between large and small (inertial) scales, and thus the introduction of turbulent eddy diffusivities $\left(v_{\mathrm{t}}\right.$ and $\left.\eta_{\mathrm{t}}\right)$. In fact, all the complexity of the nonlinear dynamics is concentrated in these coefficients. We will now try to evaluate them by going back to previous works on Alfvén wave turbulence. We first summarise previous results on anisotropic MHD turbulence. We then introduce the asymptotic equations of Alfvén wave turbulence which are not rederived here (see Galtier et al. 2000), and show their main properties. Finally, we derive the turbulent viscosities from such nonlinear equations.

\subsection{Role of anisotropy in MHD turbulence}

The first description of incompressible MHD turbulence proposed by Iroshnikov and Kraichnan (IK) in the $1960 \mathrm{~s}$ (Iroshnikov 1964; Kraichnan 1965) is based on a modified version of the Kolmogorov dimensional analysis where the largescale magnetic effects are taken into account although the fluid is supposed to be globally isotropic. The IK's heuristic model includes Alfvén waves which approximate the magnetic field at the largest scales as a uniform magnetic field. Then, it is the sporadic and successive collisions between counterpropagating Alfvén waves that produce the turbulent cascade. At leading order, the nonlinear dynamics is driven by three wave interactions, and the transfer time through the scales is estimated by $\tau_{\text {tr }} \sim \tau_{\text {nl }}^{2} / \tau_{\mathrm{A}}$, where $\tau_{\mathrm{nl}} \sim \lambda / u_{\lambda}$ is the nonlinear turnover time whereas $\tau_{\mathrm{A}} \sim \lambda / \tilde{B}_{0}$ is the Alfvén wave period (where $\tilde{B}_{0}$ stands for the Alfvén speed, taken as the rms magnetic field fluctuation). Hence, the energy spectrum is $k^{-3 / 2}$.

The weakness of the IK phenomenology is the apparent contradiction between the presence of Alfvén waves and the absence of a strong uniform magnetic field. This point and the role of a strong external magnetic field $\boldsymbol{B}_{0}$ have been widely discussed in the literature, in particular during the last two decades (Strauss 1976; Montgomery \& Turner 1981; Shebalin et al. 1983; Oughton et al. 1994; Goldreich \& Sridhar 1995; Ng \& Bhattacharjee 1996; Matthaeus et al. 1998; Galtier et al. 2000; Nazarenko et al. 2001; Milano et al. 2001; Chandran 2005; Bigot et al. 2008a). One of the most clearly established results is the bi-dimensionalization of a turbulent flow with a strong reduction of the nonlinear transfers along the $\boldsymbol{B}_{0}$ ambiant field.

In the case of coronal structures, like magnetic arcades or open magnetic fields in coronal holes, a strong magnetic field at large-scale is clearly present. This field is often modeled as a uniform field through, for example, the approximation of reduced MHD (see, e.g., Dmitruk \& Matthaeus 2003; Buchlin et al. 2007). It was thought until recently (see e.g. Oughton et al. 2004) that the reduced MHD approximation was only able to describe the regime of strong turbulence but a recent work (Nazarenko 2007) has extended its domain of application to the wave turbulence regime as well. This point is particularly important for our model which is based on the latter regime. In our model, the magnetic arcade is composed of a network of parallel strands that are maintained in a fully developed turbulent regime. Contrary to the isotropy hypothesis originally assumed by Heyvaerts \& Priest (1992), we include anisotropic effects through turbulent viscosities. In this approach, it is thus necessary to distinguish between the perpendicular $(\perp)$ and parallel $(\|)$ directions to the uniform $\boldsymbol{B}_{0}$ field. 
An important anisotropic property discussed in the literature (Higdon 1984; Goldreich \& Sridhar 1995; Galtier et al. 2005; Boldyrev 2006) is the interdependence of perpendicular and parallel scales. According to direct numerical simulations (see, e.g., Cho \& Vishniac 2000; Maron \& Goldreich 2001; Shaikh \& Zank 2007; Bigot et al. 2008b), one of the most fundamental results seems to be the (critical) balance between the nonlinear and Alfvén times which leads to the scaling relation $k_{\|} \sim k_{\perp}^{2 / 3}$. In other words, a turbulent MHD flow evolving in a strongly magnetized medium seems to be characterized by an approximately constant ratio (generally smaller than one) between the Alfvén and the nonlinear times. This result (generally) implies a dynamics mainly driven by Alfvén wave interactions.

\subsection{Alfvén wave turbulence}

The incompressible MHD Eqs. (2)-(5) may be reformulated in terms of the Elsässer fluctuations

$\boldsymbol{z}^{s}=\boldsymbol{u}+s \tilde{\boldsymbol{b}}$

with magnetic fields written in velocity units $\left(\tilde{\boldsymbol{b}}=\boldsymbol{b} / \sqrt{\mu_{0} \rho_{0}}\right)$, and in the inviscid case (i.e. in the absence of the dissipative terms proportional to the kinematic viscosity and the magnetic diffusivity),

$$
\begin{aligned}
\partial_{\mathrm{t}} z^{s}-s \tilde{\boldsymbol{B}}_{0} \cdot \boldsymbol{\nabla} z^{s} & =-z^{-s} \cdot \boldsymbol{\nabla} z^{s}-\nabla P_{*}, \\
\nabla \cdot z^{s} & =0 .
\end{aligned}
$$

Here, $s= \pm$, stands for the directional polarity, indicating the propagating direction of waves along $\tilde{\boldsymbol{B}}_{0}=\boldsymbol{B}_{0} / \sqrt{\mu_{0} \rho_{0}} ; P_{*}$ is the total (kinetic plus magnetic) pressure. Note that the present analysis is focused on the nonlinear plasma dynamics from which the turbulent transport coefficients will be derived. In the presence of a strong uniform magnetic field, MHD turbulence may be dominated by wave dynamics for which the nonlinearities are weak, and thus $\tau_{\mathrm{A}} \ll \tau_{\mathrm{nl}}$. In this limit, a small formal parameter $\epsilon$ can be introduced to measure the strength of the nonlinearities to give, for the $j$ th-component

$$
\left(\partial_{\mathrm{t}}-s \tilde{B}_{0} \partial_{\|}\right) z_{j}^{s}=-\epsilon z_{m}^{-s} \partial_{m} z_{j}^{s}-\partial_{j} P_{*}
$$

(with Einstein's notation used for indices). Note that the parallel direction $(\|)$ corresponds to the $z$-direction.

We will Fourier transform such equations, with the following definitions for the Fourier transform of the Elsässer field components $z_{j}^{s}(\boldsymbol{x}, t)$ :

$z_{j}^{s}(\boldsymbol{x}, t)=\int a_{j}^{s}(\boldsymbol{k}, t) \mathrm{e}^{\mathrm{i}\left(\boldsymbol{k} \cdot \boldsymbol{x}+s \omega_{k} t\right)} \mathrm{d} \boldsymbol{k}$,

where $\omega_{k}=\tilde{B}_{0} k_{\|}$is the Alfvén frequency. The quantity $a_{j}^{s}(\boldsymbol{k}, t)$ is the wave amplitude in the interaction representation, hence the factor $\mathrm{e}^{\mathrm{i} s \omega_{k} t}$. The Fourier transform of Eq. (48) thus is

$\partial_{\mathrm{t}} a_{j}^{s}(\boldsymbol{k})=-\mathrm{i} \epsilon k_{m} P_{j n} \int a_{m}^{-s}(\boldsymbol{\kappa}) a_{n}^{s}(\boldsymbol{L}) \mathrm{e}^{\mathrm{i} s \Delta \omega t} \delta_{\boldsymbol{k}, \boldsymbol{\kappa} \boldsymbol{L}} \mathrm{d} \boldsymbol{\kappa} \mathrm{d} \boldsymbol{L}$.

Here, $P_{j n}$ is the projector on solenoidal vectors such that $P_{j n}(\mathbf{k})=\delta_{j n}-k_{j} k_{n} / k^{2} ; \delta_{\boldsymbol{k}, \boldsymbol{\kappa} \boldsymbol{L}}=\delta(\boldsymbol{k}-\boldsymbol{\kappa}-\boldsymbol{L})$ reflects the triadic interaction, and $\Delta \omega=\omega_{\mathrm{L}}-\omega_{k}-\omega_{\kappa}$ is the frequency mixing. The appearance of an integration over wave vectors $\boldsymbol{\kappa}$ and $\boldsymbol{L}$ is directly linked to the quadratic nonlinearity of Eq. (48) (as a result of the Fourier transform of a correlation product).

Equation (50) is the compact expression of the incompressible MHD equations when a strong uniform magnetic is present.
It is the point of departure of the wave turbulence formalism which consists of writing equations for the long time behavior of second order moments. In such an asymptotic and statistical development, the time-scale separation, $\tau_{\mathrm{A}} / \tau_{\mathrm{nl}} \ll 1$, leads to the destruction of some nonlinear terms, including the fourth order cumulants, and only the resonance terms survive (Galtier et al. 2000, 2002). It allows one to obtain a natural asymptotic closure for the moment equations. In such a statistical development, the following general definition for the total (shear- plus pseudo-Alfvén wave) energy spectrum is used;

$\left\langle a_{j}^{s}(\boldsymbol{k}) a_{j}^{s}\left(\boldsymbol{k}^{\prime}\right)\right\rangle=E^{s}(\boldsymbol{k}) \delta\left(\boldsymbol{k}+\boldsymbol{k}^{\prime}\right) / k_{\perp}$,

where \langle\rangle stands for ensemble average and $k_{\perp}=\left|\boldsymbol{k}_{\perp}\right|$. In absence of helicities and in the case of an axially symmetric turbulence, the asymptotic equations simplify. For the shear-Alfvén waves, the energy spectrum is given by

$E_{\text {shear }}^{s}\left(k_{\perp}, k_{\|}\right)=f\left(k_{\|}\right) E_{\perp}^{s}\left(k_{\perp}\right)$,

where $f\left(k_{\|}\right)$is a function fixed by the initial conditions (i.e. there is no energy transfer along the parallel direction). The transverse part obeys the following nonlinear equation (the small parameter $\epsilon$ is now included in the time variable)

$$
\begin{aligned}
\partial_{\mathrm{t}} E_{\perp}^{s}\left(k_{\perp}\right)= & \frac{\pi}{\tilde{B}_{0}} \iint \cos ^{2} \phi \sin \theta \frac{k_{\perp}}{\kappa_{\perp}} E_{\perp}^{-s}\left(\kappa_{\perp}\right) \\
& \times\left[k_{\perp} E_{\perp}^{s}\left(L_{\perp}\right)-L_{\perp} E_{\perp}^{s}\left(k_{\perp}\right)\right] \mathrm{d} \kappa_{\perp} \mathrm{d} L_{\perp}
\end{aligned}
$$

where $\phi$ is the angle between $\boldsymbol{k}_{\perp}$ and $\boldsymbol{L}_{\perp}$, and $\theta$ is the angle between $\boldsymbol{k}_{\perp}$ and $\boldsymbol{k}_{\perp}$ with the perpendicular wave vectors satisfying the triangular relation $\boldsymbol{k}_{\perp}=\boldsymbol{L}_{\perp}+\boldsymbol{\kappa}_{\perp}$ (see Fig. 4). Note that from the axisymmetric assumption, the azimuthal angle integration has already been performed, and we are only left with an integration over the absolute values of the two wave numbers, $\kappa_{\perp}=\left|\boldsymbol{\kappa}_{\perp}\right|$ and $L_{\perp}=\left|\boldsymbol{L}_{\perp}\right|$. In the same way, equations can be written for pseudo-Alfvén waves which are passively advected by shear-Alfvén waves, namely

$$
\begin{aligned}
\partial_{\mathrm{t}} E_{\|}^{s}\left(k_{\perp}\right)= & \frac{\pi}{\tilde{B}_{0}} \iint \sin \theta \frac{k_{\perp}}{\kappa_{\perp}} E_{\perp}^{-s}\left(\kappa_{\perp}\right) \\
& \times\left[k_{\perp} E_{\|}^{s}\left(L_{\perp}\right)-L_{\perp} E_{\|}^{s}\left(k_{\perp}\right)\right] \mathrm{d} \kappa_{\perp} \mathrm{d} L_{\perp},
\end{aligned}
$$

with by definition

$E_{\text {pseudo }}^{s}\left(k_{\perp}, k_{\|}\right)=\tilde{f}\left(k_{\|}\right) E_{\|}^{s}\left(k_{\perp}\right)$

where $\tilde{f}\left(k_{\|}\right)$is a function fixed by the initial condition. Equations (53) and (54) - called wave kinetic equations only involve perpendicular nonlinear dynamics (with a dependence only on the perpendicular components of the wave vectors), a situation expected from previous works (Strauss 1976; Montgomery \& Turner 1981; Shebalin et al. 1983; Oughton et al. 1994; Goldreich \& Sridhar 1995; Ng \& Bhattacharjee 1996; Matthaeus et al. 1998) that showed the decrease of the parallel nonlinear transfers with the increasing strength of the external magnetic field $\tilde{\boldsymbol{B}}_{0}$.

\subsection{Anisotropic turbulent viscosities}

The main goal of this Section is the derivation of turbulent viscosities in the context of strongly anisotropic MHD turbulence. We have seen that wave turbulence leads to the inhibition of nonlinear transfers in the $\tilde{\boldsymbol{B}}_{0}$ direction, and to the bidimensional reduction of the dynamics. This result makes a strong difference to 


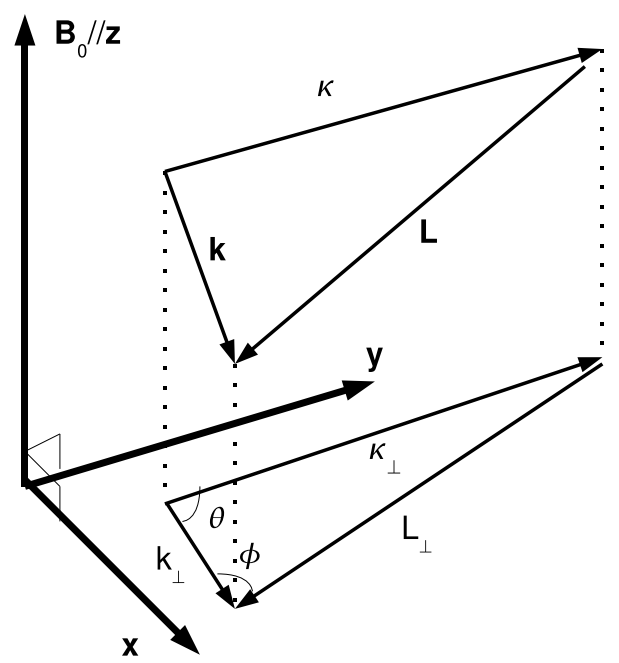

Fig. 4. Triadic interaction $\boldsymbol{k}=\boldsymbol{\kappa}+\boldsymbol{L}$ and its projection in the plane perpendicular to $\boldsymbol{B}_{0}$ (such that $\boldsymbol{k}_{\perp}=\boldsymbol{\kappa}_{\perp}+\boldsymbol{L}_{\perp}$ ).

purely isotropic MHD turbulence from which the turbulent viscosities were derived in the original model (Heyvaerts \& Priest 1992). We believe that taking into account the flow anisotropy is an important improvement in the description of the coronal heating problem.

The wave kinetic equations are assumed to describe the nonlinear dynamics at small inertial scales. These scales correspond to the scales unresolved by the current spacecrafts which are reported in Fig. 1. The turbulent viscosities represent the average effect of nonlinearities at these small scales, and they appear in the large-scale Eqs. (13), (14). In practice, we make a Taylor expansion of the wave kinetic equations for nonlocal triadic interactions (Pouquet et al. 1976) that satisfy the relation

$k_{\perp} \ll \kappa_{\perp}, L_{\perp}$,

which leads to

$L_{\perp} \simeq \kappa_{\perp}-k_{\perp} \cos \theta$,

for triadic interactions $\boldsymbol{k}_{\perp}=\boldsymbol{\kappa}_{\perp}+\boldsymbol{L}_{\perp}$ (see Fig. 4). Then, relation (57) is substituted into the kinetic Eqs. (53), (54) for, respectively, the shear-Alfvén waves

$$
\begin{aligned}
\partial_{\mathrm{t}} E_{\perp}^{s}\left(k_{\perp}\right)= & -k_{\perp}^{2} E_{\perp}^{s}\left(k_{\perp}\right) \\
& \times \int \frac{\pi}{\tilde{B}_{0}} E_{\perp}^{-s}\left(\kappa_{\perp}\right) \mathrm{d} \kappa_{\perp} \int_{0}^{2 \pi} \cos ^{2} \theta \sin ^{2} \theta \mathrm{d} \theta,
\end{aligned}
$$

and the pseudo-Alfvén waves

$$
\begin{aligned}
\partial_{\mathrm{t}} E_{\|}^{S}\left(k_{\perp}\right)= & -k_{\perp}^{2} E_{\|}^{S}\left(k_{\perp}\right) \\
& \times \int \frac{\pi}{\tilde{B}_{0}} E_{\perp}^{-s}\left(\kappa_{\perp}\right) \mathrm{d} \kappa_{\perp} \int_{0}^{2 \pi} \sin ^{2} \theta \mathrm{d} \theta .
\end{aligned}
$$

Finally, we obtain:

$\partial_{\mathrm{t}} E_{\perp}^{s}\left(k_{\perp}\right)=-v_{\perp}^{s} k_{\perp}^{2} E_{\perp}^{s}\left(k_{\perp}\right)$

$\partial_{\mathrm{t}} E_{\|}^{s}\left(k_{\perp}\right)=-v_{\|}^{s} k_{\perp}^{2} E_{\|}^{s}\left(k_{\perp}\right)$,

where $v_{\perp}^{s}$ and $v_{\|}^{s}$ are the so-called turbulent viscosities, corresponding respectively to shear- and pseudo-Alfvén waves, defined as

$$
\begin{gathered}
v_{\perp}^{s}=\frac{\pi^{2}}{4 \tilde{B}_{0}} \int E_{\perp}^{-s}\left(\kappa_{\perp}\right) \mathrm{d} \kappa_{\perp}, \\
v_{\|}^{s}=\frac{\pi^{2}}{\tilde{B}_{0}} \int E_{\perp}^{-s}\left(\kappa_{\perp}\right) \mathrm{d} \kappa_{\perp} .
\end{gathered}
$$

The turbulent viscosities are thus directly obtained after integration over the $\theta$ angle. Note that, similarly to the original paper (Heyvaerts \& Priest 1992), the integration is made from the beginning of the inertial range (at $\kappa_{i n j}$ ) where the injection of energy is made; this choice contrasts with some models where a wavenumber cut-off is chosen inside the inertial range which gives a $k$-dependence of the turbulence viscosity (see e.g. Bærenzung et al. 2008). An evaluation of the turbulent viscosities is now possible by the use of the exact power law solutions of the integro-differential Eqs. (53) and (54). This is first made in the context of a magnetic arcade for which the inward and outward Alfvén waves are exactly balanced (balanced case). This approach is then generalized to open magnetic fields for which outward Alfvén waves are dominant (unbalanced case).

\subsection{Balanced turbulence for a magnetic arcade}

For balanced turbulence, as said before, it is not necessary to distinguish outward from inward Alfvén waves. We thus drop the directional polarity $s$, and we obtain

$$
\begin{aligned}
v_{\perp} & =\frac{\pi^{2}}{4 \tilde{B}_{0}} \int E_{\perp}\left(\kappa_{\perp}\right) \mathrm{d} \kappa_{\perp}, \\
v_{\|} & =\frac{\pi^{2}}{\tilde{B}_{0}} \int E_{\perp}\left(\kappa_{\perp}\right) \mathrm{d} \kappa_{\perp} .
\end{aligned}
$$

In this case, the exact power law solution of Eq. (53) is (Galtier et al. 2000)

$E_{\perp}\left(k_{\perp}\right)=C_{\mathrm{K}} \tilde{B}_{0}^{1 / 2} P_{\perp}^{1 / 2} k_{\perp}^{-2}$

where $C_{\mathrm{K}}$ is the Kolmogorov constant whose value is about 0.585 , and $P_{\perp}$ is the constant energy flux of shear-Alfvén waves whose definition is

$\partial_{\mathrm{t}} E_{\perp}\left(k_{\perp}\right)=-\partial_{k_{\perp}} P_{\perp}\left(k_{\perp}\right)$.

It is important to note that it is the condition of constant energy flux that allows to find (by the Zakharov transformation) the exact power law solution of the nonlinear wave turbulence equation. The introduction of (66) into (64) and (65) gives

$v_{\perp}=\frac{\pi^{2}}{4} \sqrt{\frac{P_{\perp}}{\tilde{B}_{0}}} \frac{C_{\mathrm{K}}}{\kappa_{i n j}}$,
$v_{\|}=\pi^{2} \sqrt{\frac{P_{\perp}}{\tilde{B}_{0}}} \frac{C_{\mathrm{K}}}{\kappa_{i n j}}$.

Note the relation $v_{\|}=4 v_{\perp}$ between the turbulent coefficients corresponding to pseudo- and shear-Alfvén waves. In particular, this means that the pseudo-Alfvén wave energy is susceptible to decay faster than the shear-Alfvén one. Actually, this property has been detected recently in numerical simulations of freely decaying MHD turbulence (Bigot et al. 2008a). Finally note that in this wave turbulence regime, there is an automatic equipartition state between the kinetic and magnetic energies. Therefore, no distinction will be made between the average nonlinear effects acting on the velocity and on the magnetic field. A unit turbulent magnetic Prandtl number will then be taken (i.e. $v_{\mathrm{t}} / \eta_{\mathrm{t}}=1$ ).

\section{Magnetic arcade heating}

\subsection{Connection between large- and small-scale dynamics}

The magnetic arcade being supposed in a fully turbulent state, the energy transfer rate is then assumed to be constant at each 
scales in the inertial range, from the largest photospheric scales at which energy is injected, down to the small dissipative scale fom which heating starts to occur. This fundamental remark allows us to link the first estimate of the energy flux (42), based on the photospheric motions, to the flux through the anisotropic arcade scales, estimated from the turbulent eddy viscosity and magnetic diffusivity.

According to relations (52) and (67) the energy flux $P_{\perp}$, introduced above, satisfies the general relation

$\partial_{\mathrm{t}} E_{\text {shear }}\left(k_{\perp}, k_{\|}\right)=-f\left(k_{\|}\right) \partial_{k_{\perp}} P_{\perp}$.

We remind that $f$ is an arbitrary dimensionless function which reflects the absence of nonlinear transfers along the $\tilde{\boldsymbol{B}}_{0}$ direction. The coronal heating in a fraction of the arcade, say $Q$, can thus be estimated from the small-scale dynamics by

$Q=2 \ell 2 h L \rho_{0} P_{\perp} \int f\left(k_{\|}\right) \mathrm{d} k_{\|}$,

where $L$ delimits a fraction of the arcade thickness in the $y$-direction and $Q$ is measured in $\mathrm{J} \mathrm{s}^{-1}$ (note the presence of the constant mass density). In the same manner, we may evaluate the coronal heating from the large-scale energy flux $\left|\mathcal{F}_{z}\right|$. It gives for the same coronal volume:

$Q=2 h L\left|\mathcal{F}_{z}\right|$.

Hence, the relation between both photospheric and turbulent fluxes:

$P_{\perp}=\frac{\left|\mathcal{F}_{z}\right|}{2 \ell \rho_{0} \int f\left(k_{\|}\right) \mathrm{d} k_{\|}}$.

\subsection{General expression of the heating flux}

The small-scale analysis gives two turbulent viscosities coming from, respectively, the shear- and pseudo-Alfvén wave energy. To be consistent with our model, we will only consider the former one since, in the strongly anisotropic limit of wave turbulence, the pseudo-Alfvén waves fluctuate only in the parallel direction to the ambiant magnetic field. However, we note that the inclusion of the pseudo waves effect modifies only slightly the form of the turbulent viscosity (by a factor 5). Then, relation (73) gives (with $v_{\mathrm{t}} \equiv v_{\perp}$ )

$\frac{16}{\pi^{4}} v_{\mathrm{t}}^{2} \tilde{B}_{0} \frac{\kappa_{i n j}^{2}}{C_{\mathrm{K}}^{2}}=\frac{\left|\mathcal{F}_{z}\right|}{2 \ell \rho_{0} \int f\left(k_{\|}\right) \mathrm{d} k_{\|}}$.

Together with the injection wave number $\kappa_{i n j}=\pi / h$, related to the arcade thickness, we obtain

$\left|\mathcal{F}_{z}\right|=\frac{32}{\pi^{2} C_{\mathrm{K}}^{2}} \frac{v_{\mathrm{t}}^{2}}{h^{2}} \ell \rho_{0} \tilde{B}_{0} \int f\left(k_{\|}\right) \mathrm{d} k_{\|}$.

The substitution of (42) into (75) leads to the relation

$\frac{32}{\pi} \frac{v_{\mathrm{t}}^{2} \ell}{C_{\mathrm{K}}^{2} h^{3}} \int f\left(k_{\|}\right) \mathrm{d} k_{\|}=\int_{k_{i n j}}^{k_{\text {cross }}} U^{2}(k) \Psi(k) \mathrm{d} k$,

where a unit turbulent magnetic Prandtl number is taken $\left(v_{\mathrm{t}}=\right.$ $\eta_{\mathrm{t}}$ ). The above expression also gives

$\Lambda_{0} \alpha^{2} \int_{k_{i n j}}^{k_{\text {cross }}} \Psi(k) k^{-5 / 3} \mathrm{~d} k=1$, where

$\Lambda_{0}=\frac{\pi C_{\mathrm{K}}^{2}}{24}\left(\frac{\pi}{h}\right)^{5 / 3} \frac{h^{3} \bar{u}^{2}}{\ell \tilde{B}_{0}^{2}} \frac{1}{\int f\left(k_{\|}\right) \mathrm{d} k_{\|}}$.

In the next section, we use the following approximation:

$\int f\left(k_{\|}\right) \mathrm{d} k_{\|}=\pi / \ell$

\subsection{Different scale contributions}

According to the specific value of the $k$ wave number, different estimates may be made for the function $\Psi(k)$ that enters in the expression (42) of the energy injection by photospheric velocities, namely

$$
\begin{aligned}
\alpha \ll k: \Psi_{1}(k) & \simeq \frac{k}{\alpha}, \\
\sqrt{\frac{\alpha}{\ell}} \ll k \ll \alpha: \Psi_{2}(k) & \simeq 2, \\
k \ll \sqrt{\frac{\alpha}{\ell}}: \Psi_{3}(k) & \simeq \frac{\alpha}{k^{2} \ell} .
\end{aligned}
$$

We recall that $\alpha=B_{0} / \sqrt{\mu_{0} \rho_{0} v_{\mathrm{t}} \eta_{\mathrm{t}}}=\tilde{B}_{0} / \nu_{\mathrm{t}}$; it is therefore a way to measure, for example, the relative importance of the diffusive terms in Eqs. (13) and (14) which can be rewritten as

$\alpha \partial_{z} \tilde{b}(x, z)+\Delta u(x, z)=0$,

$\alpha \partial_{z} u(x, z)+\Delta \tilde{b}(x, z)=0$.

On can also more easily evaluate the relative importance of the Poynting vector (31) and the stress tensor (32) to the flux with the relation

$$
\begin{aligned}
\mathcal{P}_{z}(x, \pm \ell)+\Pi_{z}(x, \pm \ell)= & \mp \rho_{0} U(x) v_{\mathrm{t}} \\
& \times\left[\alpha \tilde{b}(x, z= \pm \ell)+\partial_{z} u(x, z= \pm \ell)\right] .
\end{aligned}
$$

For the case $\alpha \ll k$, it is clear that the main contribution to the flux comes from the stress tensor, whereas for the two other $k$ inequalities, the main contribution comes from the Poynting vector. Relation (77) can thus be written in these different regimes as:

(i) $\alpha \ll k_{\text {inj }}$,

$\Lambda_{0} \alpha^{2} \int_{k_{i n j}}^{k_{\text {cross }}} \Psi_{1}(k) k^{-5 / 3} \mathrm{~d} k=1$

(ii) $k_{i n j} \ll \alpha \ll k_{i n j}^{2} \ell$,

$\Lambda_{0} \alpha^{2}\left(\int_{k_{i n j}}^{\alpha} \Psi_{2}(k) k^{-5 / 3} \mathrm{~d} k+\int_{\alpha}^{k_{\text {cross }}} \Psi_{1}(k) k^{-5 / 3} \mathrm{~d} k\right)=1$

(iii) $k_{\text {inj }}^{2} \ell \ll \alpha \ll k_{\text {cross }}$,

$\Lambda_{0} \alpha^{2}\left(\int_{k_{\text {inj }}}^{\sqrt{\alpha / \ell}} \Psi_{3}(k) k^{-5 / 3} \mathrm{~d} k+\int_{\sqrt{\alpha / \ell}}^{\alpha} \Psi_{2}(k) k^{-5 / 3} \mathrm{~d} k\right.$
$\left.+\int_{\alpha}^{k_{\text {cross }}} \Psi_{1}(k) k^{-5 / 3} \mathrm{~d} k\right)=1 ;$ 
(iv) $k_{\text {cross }} \ll \alpha \ll k_{\text {cross }}^{2} \ell$,

$\Lambda_{0} \alpha^{2}\left(\int_{k_{\text {inj }}}^{\sqrt{\alpha / \ell}} \Psi_{3}(k) k^{-5 / 3} \mathrm{~d} k+\int_{\sqrt{\alpha / \ell}}^{k_{\text {cross }}} \Psi_{2}(k) k^{-5 / 3} \mathrm{~d} k\right)=1 ;$

(v) $k_{\text {cross }}^{2} \ell \ll \alpha$,

$\Lambda_{0} \alpha^{2} \int_{k_{i n j}}^{k_{\text {cross }}} \Psi_{3}(\alpha, k) k^{-5 / 3} \mathrm{~d} k=1$.

These relations simplify respectively to:

(i) $3 \Lambda_{0} \alpha\left(k_{\text {cross }}^{1 / 3}-k_{i n j}^{1 / 3}\right)=1$;

(ii) $3 \Lambda_{0}\left(\alpha k_{\text {cross }}^{1 / 3}+\alpha^{2} k_{\text {inj }}^{-2 / 3}-2 \alpha^{4 / 3}\right)=1$;

(iii) $3 \Lambda_{0}\left(\frac{\alpha^{3}}{8 \ell} k_{i n j}^{-8 / 3}+\frac{7}{8} \alpha^{5 / 3} \ell^{1 / 3}+\alpha k_{\mathrm{cross}}^{1 / 3}-2 \alpha^{4 / 3}\right)=1$;

(iv) $3 \Lambda_{0}\left(\frac{\alpha^{3}}{8 \ell} k_{\text {inj }}^{-8 / 3}-\alpha^{2} k_{\text {cross }}^{-2 / 3}+\frac{7}{8} \alpha^{5 / 3} \ell^{1 / 3}\right)=1$;

(v) $\frac{3}{8} \Lambda_{0} \frac{\alpha^{3}}{\ell}\left(k_{i n j}^{-8 / 3}-k_{\text {cross }}^{-8 / 3}\right)=1$.

These five different regimes correspond to a different intensity of the small-scale nonlinearities. This intensity is measured in terms of an equivalent wave number $\alpha$ and it is compared to the two available scales, namely $k_{i n j}$ and $k_{\text {cross }}$. Case (i) is the situation where the small-scale nonlinearities are the strongest: they are felt beyond $k_{i n j}$. Case (v) is the situation where the small-scale nonlinearities are the weakest: the diffusive terms in Eqs. (83), (84) are then negligible.

\subsection{Predictions for a coronal plasma}

We define the following basic coronal quantities:

$$
\begin{aligned}
h & =10^{6} \mathcal{H} \mathrm{m}, \\
\ell & =10^{7} \mathcal{L} \mathrm{m}, \\
B_{0} & =10^{-2} \mathcal{B}_{0} \mathrm{~T}, \\
\bar{u} & =10^{3} \mathcal{U} \mathrm{m} \mathrm{s}^{-1}, \\
\rho_{0} & =10^{-12} \mathcal{M ~ k g ~ m}^{-3},
\end{aligned}
$$

which give the estimates:

$$
\begin{aligned}
\tilde{B}_{0} & =8.9 \times 10^{6} \mathcal{B}_{0} \mathcal{M}^{-1 / 2} \mathrm{~m} \mathrm{~s}^{-1}, \\
k_{\text {inj }} & =3.1 \times 10^{-6} \mathcal{H}^{-1} \mathrm{~m}^{-1}, \\
k_{\text {cross }} & =2.4 \times 10^{-4} \mathcal{H}^{1 / 2} \mathcal{L}^{3 / 2} \mathcal{U}^{3 / 2} \mathcal{B}_{0}^{-3 / 2} \mathcal{M}^{-3 / 4} \mathrm{~m}^{-1}, \\
\Lambda_{0} & =1.2 \times 10^{-1} \mathcal{H}^{4 / 3} \mathcal{U}^{2} \mathcal{B}_{0}^{-2} \mathcal{M ~ m}^{4 / 3} .
\end{aligned}
$$

With these values, the only satisfied equation is (93) which corresponds to the inequality of case (iv). The solution of Eq. (93) is:

$$
\alpha=4.1 \times 10^{-3} \mathcal{H}^{-4 / 3} \mathcal{B}_{0}^{2 / 3} \mathcal{M}^{-1 / 3} \mathcal{U}^{-2 / 3} \mathrm{~m}^{-1} .
$$

Then, it gives the following estimate for the turbulent viscosity:

$v_{\mathrm{t}}=2.2 \times 10^{9} \mathcal{H}^{4 / 3} \mathcal{U}^{2 / 3} \mathcal{B}_{0}^{1 / 3} \mathcal{M}^{-1 / 6} \mathrm{~m}^{2} \mathrm{~s}^{-1}$.

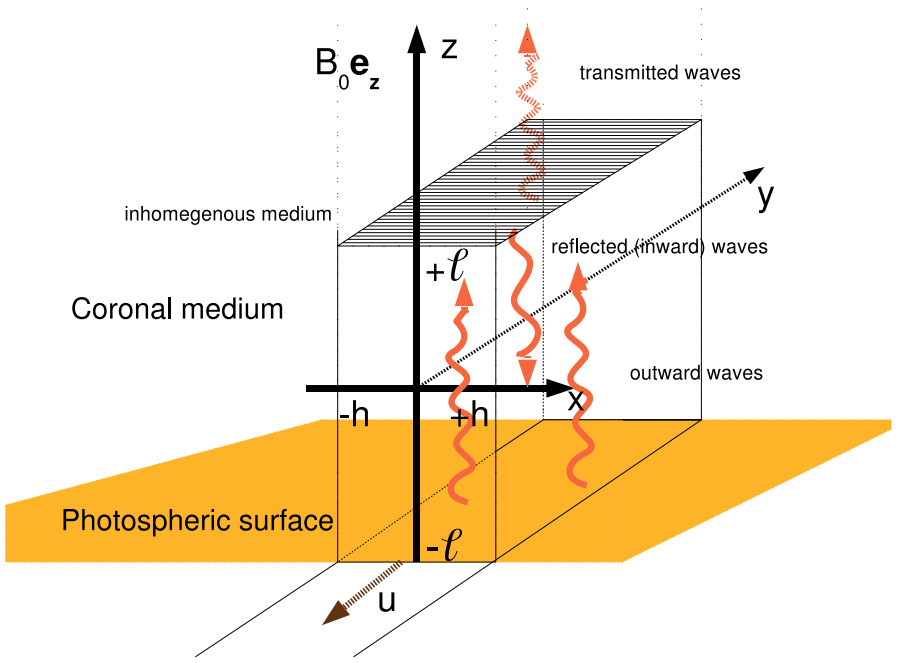

Fig. 5. Coronal hole geometry: the coronal plasma is confined in a volume delimited by $-h<x<+h$ and $-\infty<y<+\infty$, and may escape from the low coronal medium at the top of the structure (for $z>\ell$ ). The photosphere appears as a (single) lower boundary plane at altitude $z=-\ell$. The interplanetary inhomogeneity allows the reflection of a fraction of outward Alfvén waves which produces inward waves.

Hence, the heating flux per unit area

$\left|\mathcal{F}_{z}\right|=1249 \mathcal{H}^{2 / 3} \mathcal{U}^{4 / 3} \mathcal{B}_{0}^{5 / 3} \mathcal{M}^{1 / 6} \mathrm{~J} \mathrm{~m}^{-2} \mathrm{~s}^{-1}$.

The turbulent velocity of the coronal plasma may be found from relation

$u_{\perp}^{2}=\frac{\pi}{h} \int_{k_{i n j}}^{+\infty} C_{\mathrm{K}} \tilde{B}_{0}^{1 / 2} P_{\perp}^{1 / 2} k_{\perp}^{-2} \mathrm{~d} k_{\perp}$

Only the perpendicular fluctuating velocity is taken into account since we are only concerned with shear-Alfvén waves in a strongly anisotropic turbulence. We substitute $P_{\perp}$ by its expression (73) to finally obtain the following prediction:

$u_{\perp}=50 \mathcal{H}^{1 / 2} \mathcal{U}^{1 / 3} \mathcal{B}_{0}^{2 / 3} \mathcal{M}^{-1 / 6} \mathcal{L}^{-1 / 2} \mathrm{~km} \mathrm{~s}^{-1}$.

These results compare favorably with observations, in particular, in the quiet corona with a heating flux large enough to explain the observations. Note that the heating prediction is mostly sensitive to the magnetic field intensity $\mathcal{B}_{0}$ which has the larger power law index by $5 / 3$. Thus, $\mathcal{B}_{0}=3-4\left(B_{0}=3-4 \times 10^{-2} \mathrm{G}\right)$ leads to a factor about ten times larger for the heating flux with a value close to the measurements $10^{4} \mathrm{~J} \mathrm{~m}^{-2} \mathrm{~s}^{-1}$ for active regions.

\section{Coronal hole heating}

\subsection{Geometry of open magnetic field lines}

In this section, the model prediction for the heating rate is extended to coronal holes where the plasma is guided along a large-scale magnetic field which expands into the interplanetary medium. It is along such structures (mainly at the poles) that the fast solar wind is released whereas the slow wind is freed at lower latitudes around the equatorial plane. In these configurations of open magnetic lines, the reflection of outward Alfvén waves, due to some inhomogeneities, produces inward waves which eventually sustain nonlinear interactions. In this case, the coronal heating is clearly dependent on the reflection rate of 
Alfvén waves (Velli et al. 1993; Dmitruk \& Matthaeus 2003). The precise origin of the partial reflection of Alfvén waves is still under debate. Therefore, any theoretical prediction is useful for the comparison between observations and models, and eventually for the understanding of the solar corona dynamics. In this Section, we seek theoretical predictions like the reflection rate needed to sustain an efficient coronal heating localized in coronal holes. We see that a small reflection rate of $15-20 \%$ is enough to recover the coronal heating observations. This result may constrain the efficiency of the mechanisms invoked to produce reflected Alfvén waves.

Figure 5 shows a schematic view of open magnetic field lines which only differs from the magnetic loop configuration (Fig. 3) is the upper boundary condition. This second photospheric surface is replaced by a permeable boundary from which outward Alfvén waves may be partially reflected. Note that the first model of magnetic arcades corresponds to the case where outward Alfvén waves are totally reflected and where outward and inward waves are balanced.

\subsection{Reflection rate versus cross-helicity}

To quantify the reflection rate of Alfvén waves (also called the Alfvénicity), a characteristic quantity is the normalized crosshelicity $\sigma_{\mathrm{c}}$ defined as

$\sigma_{\mathrm{c}}=\frac{z^{+2}-z^{-2}}{z^{+2}+z^{-2}}$,

where $z^{+}$is the Alfvén inward wave and $z^{-}$is the outward wave (with $z^{s}=\left|z^{s}\right|, s= \pm$ ). Such a quantity provides a measure of the relative importance between outgoing and ingoing Alfvén waves. In particular, $\sigma_{\mathrm{c}}= \pm 1$ involves the presence of only one type of polarity, and consequently, the absence of nonlinear interactions (thus no heating at dissipative scales), whereas a balance between $z^{+}$and $z^{-}$waves implies $\sigma_{\mathrm{c}}=0$ leading to more important nonlinear interactions (between waves of different polarity) as was assumed in the magnetic arcade (loop) configuration analyzed in previous sections. The reduced cross-helicity is indeed related to the wave reflection rate, $\mathcal{R}$, such as

$\mathcal{R}=\frac{1-\left|\sigma_{\mathrm{c}}\right|}{1+\left|\sigma_{\mathrm{c}}\right|}$.

The case $\mathcal{R}=1$ (all outward waves are reflected) is similar to the loop configuration in which there is the same number of inward and outward waves, whereas in the case of $\mathcal{R}=0$ no waves are reflected.

\subsection{Unbalanced turbulence for coronal holes}

The integro-differential kinetic Eqs. (53) for the Alfvén waves are numerically integrated with a logarithmic subdivision of the $k$ axis : $k_{i}=\delta k 2^{i / F}$, where $i$ is an integer $(i=1, N), \delta k=0.125$ is the smallest wave number reached in the computation, and $F=8$ is the refinement of the grid. (This method was previously performed and optimized in Galtier et al. 2000.) This numerical technique allows to reach larger Reynolds numbers since, for a wave number resolution $N=157$, the maximum wave number reached, $k_{\max }$, is around $10^{5}$ (the magnetic Prandtl number is equal to unity). Note that dissipative terms are introduced in the inviscid kinetic equations to avoid numerical instabilities, and the viscosity is fixed to $10^{-5}$. The initial Elsässer fields are injected in the wave number range $[\delta k, 44]$ with an energy spectrum proportional to $k_{\perp}^{3} \exp \left(-k_{\perp}^{2} / 2\right)$, peaking at $k_{\perp_{0}} \sim 1$. The

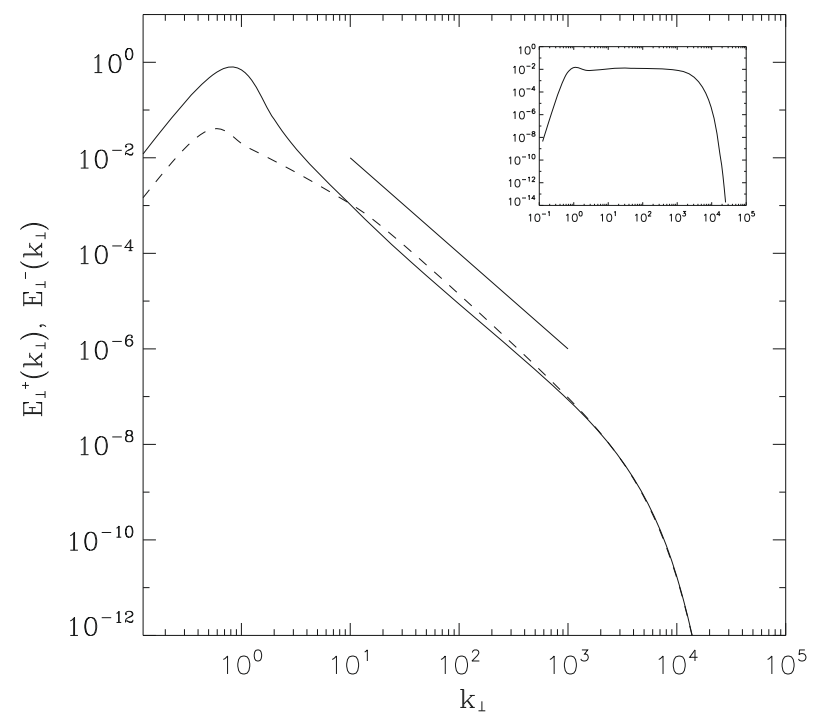

Fig. 6. Energy spectra of shear-Alfvén waves $E_{\perp}^{+}$(solid line) and $E_{\perp}^{-}$ (dashed line) with $\mathcal{R}=25 \%\left(\sigma_{\mathrm{c}}=0.8\right)$. The straight line follows a $k_{\perp}^{-2}$ law which is the theoretical prediction for the balanced turbulence case ( $\mathcal{R}=100 \%$ and $\sigma_{\mathrm{c}}=0$ ). Inset: compensated product of energy spectra as $E_{\perp}^{+} E_{\perp}^{-} k_{\perp}^{4}$.

corresponding $U_{\text {rms }}$ and integral scale $L_{0}$ are, respectively, about 4.75 and 1.5, which gives an initial Reynolds number $\left(U_{\mathrm{rms}} L_{0} / v\right)$ of about $10^{6}$. The flow is then left to freely evolve.

In Fig. 6, we show instantaneous energy spectra of the shearAlfvén waves, $E_{\perp}^{+}$and $E_{\perp}^{-}$, obtained for, respectively, $z_{\perp}^{+}$and $z_{\perp}^{-}$ fluctuations with a cross-helicity of $0.8(\mathcal{R}=25 \%)$. (In the following we respectively consider $z_{\perp}^{-}$and $z_{\perp}^{+}$as the inward (reflected) and outward waves) The chosen time is the one at which the energy spectra are the most developed, i.e. the most extended towards large wave numbers $k_{\perp}$. Different behaviors are clearly found for the $E_{\perp}^{+}$and $E_{\perp}^{-}$spectra with, at large scales, a domination of inward Alfvén waves linked to the choice of the initial conditions. At smaller scales, we see the appearance of an extended inertial range where the inward waves $\left(E_{\perp}^{-}\right)$slightly dominate. Note that the energy spectra product, $E_{\perp}^{+} E_{\perp}^{-}$, follows a $k_{\perp}^{-4}$ scaling law as predicted theoretically (Galtier et al. 2000), and shown in the inset of Fig. 6. Finally the two energy spectra overlap at dissipative scales. From such a numerical simulation, we are able to compute the turbulent viscosities for unbalanced turbulence by integration of the spectra over wave numbers, from the beginning of the inertial range up to the dissipative scales. This computation is done for several simulations corresponding to different reflection rates.

\subsection{Parametric study and predictions}

A parametric study of the heating flux according to the reflection rate of outward waves is performed. The turbulent viscosities are calculated for different values of the reflection rate by integrating the shear-Alfvén wave spectra, from the injection scale (normalized to unity in the numerical simulation) up to the dissipative scales, and by using relation (62) to estimate $v_{\perp}^{s}$. In such a calculation, the chosen time is the one at which the energy spectra are most developed. Since the result depends on the initial amount of energy taken in the simulation, the flux is normalized to the flux obtained in the loop case (see Sect. 4) for which a balanced turbulence is assumed. With this method, we are able 


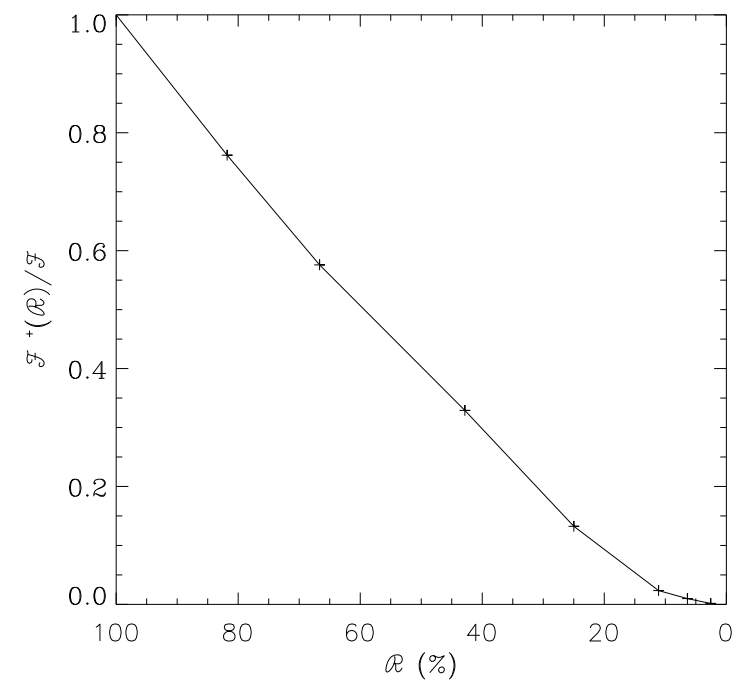

Fig. 7. Evolution of the heating flux according to the reflection rate of outward Alfvén waves. The heating flux is normalized to the flux obtained in the balanced turbulence case (Sect. 4), i.e. for $\mathcal{R}=100 \%$.

to approximately predict the variation of the relative heating flux as a function of the reflection rate.

From the turbulent flux expression (75), we obtain the relations

$\frac{\left|\mathcal{F}_{z}^{s}(\mathcal{R})\right|}{\left|\mathcal{F}_{z}\right|} \simeq\left(\frac{v^{s}(\mathcal{R})}{v_{\perp}}\right)^{2}=\frac{\left[\int E_{\perp}^{-s}(\mathcal{R}) \mathrm{d} \kappa_{\perp}\right]^{2}}{\left[\int E_{\perp} \mathrm{d} \kappa_{\perp}\right]^{2}}$

which depend on the reflection rate of outward waves $\mathcal{R} . \mathcal{F}_{z}^{s}$ and $\mathcal{F}_{z}$ are, respectively, the heating fluxes in the unbalanced and balanced turbulent cases. The presence of the directional polarity $s= \pm$ keeps track of the advection between waves of different polarity. The calculation made here concerns the heating flux at the lower boundary $(z=-\ell)$ since the upper boundary condition previously used is no longer the same. We have also assumed, in particular, that the Kolmogorov constant does not change drastically for different reflection rates. Figure 7 shows the flux ratio as a function of the reflection rate $\mathcal{R}$. As expected, the curve decreases with the reflection rate, meaning that the turbulent heating is clearly less efficient for smaller values of $\mathcal{R}$. Finally, the flux ratio goes to zero when only one type of wave remains (since in this case the nonlinear interactions disappear). Note that the decreasing function is close to a linear curve with a flux ratio of about $40 \%$ for $\mathcal{R}=50 \%$.

The heating flux prediction in coronal holes is known and estimated as around $100 \mathrm{~J} \mathrm{~m}^{-2} \mathrm{~s}^{-1}$ (Withbroe et al. 1977). Since the background magnetic field is still about $10^{-2} \mathrm{~T}$, we may find the reflection rate in coronal holes by using both the relation (106) and the curve in Fig. 7. The value $\mathcal{R}=25 \%$ leads to a flux ratio of about $15 \%-10 \%$, a small enough value to recover the heating flux for coronal holes (without taking into account the fast solar wind). We see that a relatively small reflection rate is therefore enough to produce an efficient coronal heating due to anisotropic MHD turbulence.

\section{Discussion and conclusion}

In this paper, we have developed an analytic model for strongly anisotropic structures in order to recover well known coronal heating rates for the quiet sun, active regions and coronal holes. The coronal structures are assumed to be in a turbulent state maintained by the slow motion of the magnetic footpoints anchored on the photospheric surface. The main difficulty is that existing spacecraft are unable to resolve all the inertial (small) scales, and a fortiori the dissipative scales. So, firstly, the injected energy at large scale is resolved from incompressible MHD equations, and for specific boundary conditions satisfying the divergence free condition. Secondly, the (unresolved) smallscale dynamics is modeled by turbulent viscosities derived from an asymptotic (exact) closure model of wave turbulence for the case of the loop configuration (also called balanced turbulence), i.e. when there are as many inward as outward waves which nonlinearly interact. In the open magnetic line configuration the nonlinear interactions are sustained by the conversion of outward waves into inward waves by reflection (unbalanced turbulence). We have numerically integrated the kinetic equation of Alfvén waves for different values of the reflection rate, and plotted the evolution of the coronal heating flux according to this rate, as a measure of the relative magnitude of ingoing and outgoing waves. Finally these results are compared to the balanced turbulence flux (loop configuration).

For standard loop geometry parameters, and for a magnetic field intensity of $B_{0}=10^{-2} \mathrm{~T}$, we find a heating flux prediction of about $1.2 \times 10^{3} \mathrm{~J} \mathrm{~m}^{-2} \mathrm{~s}^{-1}$ which is close to the value measured in the quiet sun $\left(10^{3} \mathrm{~J} \mathrm{~m}^{-2} \mathrm{~s}^{-1}\right)$. Moreover, the prediction for the turbulent velocity $\left(50 \mathrm{~km} \mathrm{~s}^{-1}\right)$ compares favorably with the measurements of nonthermal velocities $\left(30 \mathrm{~km} \mathrm{~s}^{-1}\right)$ in the quiet solar corona, as well as with the maximal values of some line profiles $\left(55 \mathrm{~km} \mathrm{~s}^{-1}\right)$ found by the SUMER instrument (Chae et al. 1998). For active regions, our estimation tends toward a value of about $10^{4} \mathrm{~J} \mathrm{~m}^{-2} \mathrm{~s}^{-1}$ for a magnetic field intensity stronger (3-4 times larger) than for the quiet sun, which is an expected value for strong solar activity. For the heating rate prediction in open magnetic lines, we take as a reference the loop configuration (for which there is a balanced turbulence) and the same parameters as for the quiet sun. We can recover the well known estimates for coronal holes of about $100 \mathrm{~J} \mathrm{~m}^{-2} \mathrm{~s}^{-1}$ (without taking into account the fast solar wind) for a reflection rate of outward waves of about $15-20 \%$.

The previous predictions do not take into account the coronal heating by pseudo-Alfvén waves (nor the heating due to the pure $2 \mathrm{D}$ state - with wave vectors such as $\left(\boldsymbol{k}_{\perp}, k_{\|}=0\right)$ - which is not described by Alfvén wave turbulence), since their parallel fluctuations are excluded by the boundary conditions (involving only perpendicular fluctuations). Thus, their insertion should heat slightly more the solar corona and raise our predictions slightly. Moreover, in the computation of heating flux for the unbalanced turbulence case, only the heating due to outward Alfvén waves is considered. The heating due to inward waves should modify slightly the heating prediction for coronal holes, and therefore allows a slight decrease of the reflection rate with a heating rate maintained around at $100 \mathrm{~J} \mathrm{~m}^{-2} \mathrm{~s}^{-1}$.

Acknowledgements. Financial support from PNST/INSU/CNRS are gratefully acknowledged. This work was supported by the ANR project no. 06-BLAN0363-01 "HiSpeedPIV". We thank the anonymous referee for comments and questions which have improved the presentation of the paper.

\section{References}

Alfvén, H. 1947, MNRAS, 107, 211

Bærenzung, J., Politano, H., Ponty, Y., \& Pouquet, A. 2008, Phys. Rev. E, 77, 046303

Bigot, B., Galtier, S., \& Politano, H. 2008a, Phys. Rev. Lett., 100, 074502

Bigot, B., Galtier, S., \& Politano, H. 2008b, Phys. Rev. E, to be published [arXiv: 0808.3061v1] [physics . flu-dyn]

Buchlin, E., Cargill, P., Bradshaw, S. J., \& Velli, M. 2007, A\&A, 469, 347 
Chae, J., Schuhle, U., \& Lemaire, P. 1998, ApJ, 505, 957

Chandran, B. D. G. 2005, Phys. Rev. Lett., 95, 265004

Chou, D.-Y., LaBonte, B. J., Braun, D. C., \& Duvall, Jr., T. L. 1991, ApJ, 372, 314

Dmitruk, P., \& Gomez, D. O. 1997, ApJ, 484, L83

Dmitruk, P., \& Matthaeus, W. H. 2003, ApJ, 597, 1097

Dmitruk, P., Milano, L. J., \& Matthaeus, W. H. 2001, ApJ, 548, 482

Doschek, G. A., Mariska, J. T., Warren, H. P., et al. 2007, ApJ, 667, L109

Einaudi, G., Velli, M., Politano, H., \& Pouquet, A. 1996, ApJ, 457, L113

Espagnet, O., Muller, R., Roudier, Th., \& Mein, N. 1993, A\&A, 271, 589

Galtier, S., \& Pouquet, A. 1998, Sol. Phys., 179, 141

Galtier, S., \& Bhattacharjee, A. 2003, Phys. Plasmas, 10, 3065

Galtier, S., Nazarenko, S. V., Newell, A. C., \& Pouquet, A. 2000, ApJ, 63, 447

Galtier, S., Nazarenko, S. V., Newell, A. C., \& Pouquet, A. 2002, J. Plasma Phys., 564,49

Galtier, S., Mangeney, A., \& Pouquet, A. 2005, Phys. Plasmas, 12, 092310

Gomez, D. O.. \& Ferro Fontan, C. 1988, Sol. Phys., 116, 33

Gomez, D. O., \& Ferro Fontan, C. 1992, ApJ, 394, 662

Goldreich, P., \& Sridhar, S. 1995, ApJ, 438, 763

Heyvaerts, J., \& Priest, E. R. 1983, A\&A, 117, 220

Heyvaerts, J., \& Priest, E. R. 1992, ApJ, 390, 297

Hollweg, J. V. 1984, ApJ, 277, 392

Higdon, J.-C. 1984, ApJ, 285, 109

Inverarity, G. W., \& Priest, E. R. 1995, A\&A, 296, 396

Iroshnikov, P. S. 1964, Sov. Astron., 7, 566
Kraichnan, R. H. 1965, Phys. Fluids, 8, 1385

Matthaeus, W. H., Oughton, S., Ghosh, S., \& Hossain, M. 1998, Phys. Rev. Lett., 81,2056

Milano, L. J., Gomez, D. O., \& Martens, P. C. H. 1997, ApJ, 490, 442

Milano, L. J., Matthaeus, W. H., Dmitruk, P., \& Montgomery, D. C. 2001, Phys. Plasmas, 8, 2673

Montgomery, D., \& Turner, L. 1981, Phys. Fluids, 24, 825

Nature 2007, Nature, 446, 477

Nazarenko, S. V. 2007, New J. Phys., 9, 307

Nazarenko, S. V., Newell, A. C., \& Galtier, S. 2001, Physica D, 152, 646

Ng, C. S., \& Bhattacharjee, A. 1996, ApJ, 465, 845

Oughton, S., Priest, E. R., \& Matthaeus, W. H. 1994, J. Fluid Mech., 280, 95

Oughton, S., Dmitruk, P., \& Matthaeus, W. H. 2004, Phys. Plasmas, 11, 2214

Pouquet, A., Frisch, U., \& Leorat, J. 1976, J. Fluid Mech., 77, 321

Priest, E. R., \& Forbes, T. G. 2000 (Cambridge University Press)

Roudier, Th., \& Müller, R. 1987, Sol. Phys., 107, 11

Saur, J., Politano, H., Pouquet, A., \& Matthaeus, W. H. 2002, A\&A, 386, 699

Shaikh, D., \& Zank, G. 2007, ApJ, 656, L17

Shebalin, J. V., Matthaeus, W. H., \& Montgomery, D. 1983, J. Plasma Phys., 29, 525

Strauss, H. R. 1976, Phys. Fluids, 19, 134

Velli, M. 1993, A\&A, 270, 304

Verdini, A., \& Velli, M. 2007, ApJ, 662, 669

Warren, H. P., Mariska, J. T., Wilheim, K., \& Lemaire, P. 1997, ApJ, 484, 91

Withbroe, G. L., \& Noyes, R. W. 1977, ARA\&A, 15, 363 